\title{
Fast-track surgery and enhanced recovery after surgery concepts after their application in hernia repair
}

\author{
V.V. Boiko ${ }^{1}$, K.Y. Parkhomenko², O.E. Feskov ${ }^{3}$, A.Y. Gavrikov ${ }^{3}$, S.V. Kursov ${ }^{3}$, V.I. Piatnochka ${ }^{4}$ \\ 1"V.T. Zaitsev" Institute of General and Emergency Surgery, \\ Academy of Medical Sciences of Ukraine, Kharkiv, Ukraine \\ ${ }^{2}$ Family Medicine and Internal Medicine Department, Kharkiv National Medical University, Kharkiv, Ukraine \\ ${ }^{3}$ Emergency Medicine and Disaster Medicine Department, \\ Kharkiv Medical Academy of Postgraduate Education, Kharkiv, Ukraine \\ ${ }^{4}$ Department of Surgery, Institute of Postgraduate Education, \\ "I. Horbachevsky" Ternopil National Medical University, Ternopil, Ukraine
}

\begin{abstract}
The growing number of surgical interventions that occur worldwide, along with the increasing prevalence of general somatic pathology, keeps current the problem of preventing complications during surgery and in the postoperative period. An efficient solution to this problem is to determine the risk of surgery, directed preoperative preparation, taking into account the comorbidity, optimization of surgery and postoperative rehabilitation. Fast-track surgery and enhanced recovery after surgery programs have significant potential for improving outcomes of surgery. These programs are widely used in various fields of surgery, but their effectiveness in herniology, especially in the performing simultaneous surgeries, there are very few reports, and the results of individual studies are contradictory. The review provides an analysis of holistic strategies of fast-track surgery and enhanced recovery and individual measures to assess the effectiveness of their impact on the immediate results of surgical interventions.
\end{abstract}

Keywords: fast-track surgery, enhanced recovery after surgery, multimodal anesthesia, ventral hernia repair, immediate result

\section{INTRODUCTION}

Surgery for many diseases is a mandatory or optional method of treatment. According to a global study, the total number of surgeries in 2012 was 359.5 million, which is $38 \%$ more than in 2004 [1]. At the same time, even after elective surgeries, the frequency of complications reaches $16.8 \%$, and postoperative mortality $-0.5 \%$ [2]. Postoperative complications are the cause of death, long-term or permanent deterioration of health and huge material losses of national health systems. Most publications on surgical topics are devoted to the prevention of early and late complications after various surgeries. Multiple measures of preoperative preparation, improvements in methods of surgical interventions, intraoperative and postoperative management of patients were offered. But a really revolutionary approach was the similar strategy of fast-track surgery (FTS) and enhanced recovery after surgery (ERAS).

\section{COMPREHENSIVE STRATEGIES IN IMPROVING THE SURGICAL OUTCOMES}

FTS and ERAS strategies are relatively new concepts of surgical care for patients based on an understanding of the pathophysiology of postaggressive responses to surgery and other nondrug and drug interventions. The term fast-track surgery can be defined as a coordinated perioperative approach to the treatment of the patient, which aims to maximize the intensity of surgical stress 
and recovery of the patient as soon as possible after surgery [3]. The main principle of fast-track surgery is the treatment of the disease with minimal disruption of the patient's physiology using evidence-based strategies to accelerate recovery after surgery. The combination of the best surgical methods, modern anesthesia and pain control methods is aimed at reducing the perioperative response to stress and organ dysfunction, the frequency of postoperative complications, along with reducing the cost and duration of inpatient treatment $[4,5]$. ERAS strategy is focused mainly on optimizing of postoperative management of patients, but with the use of similar multimodal principles. This method is also used in the surgery of ventral hernias [6].

The consistent concept of fast-track surgery and enhanced recovery applies to all stages of perioperative care with a large number of different measures in the preoperative period, during surgery and in the postoperative period [4,7]. Preoperative assessment allows determining the risks and the possibility of stabilizing the comorbidity and optimizing the function of the organs before surgery. Comorbidity increases the risk of complications and mortality during abdominal wall reconstruction, so the protocols of enhanced recovery should take into account preoperative improvement of the condition [8]. Careful preoperative preparation of patients in whom hernioplasty is planned, allows preventing postoperative complications [9]. In addition, patients receive information about the planned course of postoperative tretment, analgesia, mobilization and activation program. Several studies have shown that such information can reduce anxiety, the need for narcotic analgesics, reduce or eliminate the side effects associated with their use, and length of hospital stay [10-12]. K. Slim and D. Standaert (2020) believe that in patients with postoperative ventral hernias $(\mathrm{PVH})$, due to comorbidity, pre-rehabilitation with smoking cessation, treatment of obesity or excessive weight loss and diabetes mellitus (DM) is the most important in enhanced recovery programs. But the level of evidence in patients with PVH is not yet sufficient [13,14].

In most programs of enhanced recovery after surgery, when choosing the method of surgical intervention, minimally invasive methods are preferred, in particular, with the use of video laparoscopic techniques. Open hernioplasty is associated with a significant amount of surgical trauma, as well as a greater expactancy of systemic inflammatory response, significant stress on humoral mechanisms, greater manifestations of pain, greater need for analgesics [15-17].

However, in many cases it is not possible to abandon open surgeries. Shyam D.C. and Rapsang A.G. (2013) reported that «open» surgeries can be used in patients of older age groups. Despite the fact that the use of «open» technology is associated with longer hospital stays, the mortality rate is zero, and the complication rate and recurrence rate is very low, even in those with concomitant diseases [18]. Nanavati A.J. and Prabhakar S. (2014) in the analysis of the frequency of use of "open" and laparoscopic techniques in fast-track surgery found that most clinical studies that examined the fasttrack procedures involved the liberal use of laparoscopic methods. However, when the protocol of optimal perioperative care was used for open surgeries, no significant difference was found between the results of treatment [19]. It should also be noted that the use of laparoscopic technique is associated with certain complications, the probability of which is higher in elderly and senile patients and in the presence of serious comorbidity [20]. Patients with reduced cardiovascular and respiratory function, including chronic heart failure (CHF), chronic obstructive pulmonary disease (COPD), emphysema, and pneumosclerosis have an increased risk of hypoxemia during laparoscopic procedures [21].

The use of a combination of fast-track rehabilitation methods can affect the outcome more than the choice between laparoscopic technique and «open» surgery. The results of some studies indicate that within the emergency surgery program there is no difference between laparoscopic and open surgery in terms of postoperative recovery or duration of hospital stay [22, 23]. On the other hand, the results of the analysis of the effectiveness of enhanced recovery programs and their components showed that the improvement of results was associated with the most complete implementation of the protocol, and among the individual components had a significant impact only minimally invasive procedure $(\mathrm{OR}=0.63 ; 95 \%$ CI - 0.4-0.9), which led to a decrease in the duration of hospitalization and the frequency of severe complications [24].

One of the reasons for the adverse consequences of surgical interventions is inadequate preoperative preparation. Traditional bowel preparation showed to be potentially harmful, increasing the risk of sepsis and worsening postoperative dehydration. Instead of long-term refusal from oral food and fluid intake, it is allowed to drink up to 
$150 \mathrm{ml}$ orally 2 hours before surgery to quench thirst. Additional intravenous administration to patients for a night before the planned surgery, as well as one hour before the start of $5 \%$ glucose solution with electrolytes significantly increases the patient's comfort, relieving thirst, eliminating hunger and anxiety $[25,26]$.

In the complex of emergency surgery, an important measure is to abandon the routine use of nasogastric tubes and drainage of the abdominal cavity. It was found that they rather increase the complications of the postoperative period and prolong the hospital stay $[27,28]$. Abdominal drainage does not prevent the development of complications but, on the contrary, is more often associated with the development of postoperative ileus [29-31].

Adequate postoperative nutrition is very important. The oral way of administration is traditionally limited in the postoperative period and involves a gradual transition from fluid intake to solid foods. However, several studies have shown that early eating is safe even after bowel resection because early enteral nutrition reduces intestinal permeability, which also reduces infection by reducing bacterial translocation. Rapid recovery of peristalsis is facilitated by epidural anesthesia, refusal or reduction of opioid dose, normalization of water-salt metabolism and targeted drug therapy [32$35]$. In the last decade, in order to prevent disorders of motor-evacuatory function of the gastrointestinal tract, Alvimopan has been used, which is an antagonist of peripheral $\mu(\mathrm{mu})$-opioid receptors and slows down the inhibitory effect of opioid analgesics on the gastrointestinal tract [3638]. Another extremely "fashionable" way to solve the problem of postoperative ileus removal is the use of chewing gum by patients undergone surgery. Chewing gum promotes early recovery of intestinal function after abdominal surgery, which explains the increase in the concentration of gastrin, neurotensin neuropeptide, pancreatic polypeptides with increased secretion of the duodenum and increased myoelectric activity of the intestine [39-41].

Enhanced recovery of complete nutrition makes it possible to optimize fluid supply. Excessive parenteral hydration can lead to lung and heart dysfunction, impair wound healing due to edema and reduced tissue oxygenation. The accumulation of fluid in the intestinal interstitium leads to an increase in intra-abdominal pressure (IAP), helps to limit pulmonary ventilation, circulatory disorders in the intestinal wall and restricts renal blood flow. Therefore, the management of infusion therapy should be arranged for each patient $[42,43]$.
Early mobilization of the patient is a universal component of any enhanced recovery protocols. Prolonged bed rest increases muscle loss, contributes to hypoventilation, lung dysfunction, infections and thromboembolic complications. Early mobilization of patients instead reduces fatigue, improves sleep, accelerates the return to leisure activities and significantly reduces the risk of the negative effects mentioned above [44-46].

Multimodal analgesia during surgery and in the postoperative period is of particular importance in enhanced recovery programs. Postoperative pain exacerbates the surgical response to stress, organ dysfunction, and delays recovery. Severe stress and active use of opioids make it difficult to restore full gastrointestinal function and delay the activation of patients [47-49]. Therefore, multimodal analgesia is used in surgeries associated with subsequent intense pain. The principle of multimodal or balanced analgesia is to obtain additional effects from various forms of pain control while minimizing side effects, such as sedation, nausea, ileus and urinary retention. Multimodal analgesia combines several strategies. First of all, it is the rejection of opioids and the use of non-opioid drugs, which include nonsteroidal anti-inflammatory drugs (NSAIDs), glutamate antagonists (magnesium, ketamine), GABAergic measures (gabapentin), agonists of $\alpha 2$-adrenergic receptor as well as methods of regional anesthesia [50-52]. Due to the use of NSAIDs, a powerful anti-inflammatory effect is achieved, which after surgery provides a significant reduction in the severity of pain and significantly reduces its duration, which contributes to early motor activation of patients after surgery $[53,54]$. Reducing the need for opioids accelerates the restoration of cognitive functions, effective self-breathing, absorption capacity of the stomach and intestinal wall, provides a rapid reduction in IAP, and at the same time increases and accelerates blood flow in the intestinal wall and renal blood flow $[22,55]$.

In addition, in many cases, attempts are made to limit the use of general anesthesia through the widespread use of regional anesthesia, including spinal and epidural anesthesia, transversus abdominis plane block (TAP-block) techniques, and blockade of nerve trunks and vaults prolonged catheterization [56]. In patients with inguinal hernias, paravertebral block, as the main method of anesthesia or as a supplement to general anesthesia, contributed to reduce the frequency and severity of urinary retention after hernioplasty and provided good anesthesia [57]. Blockade of the 
transversus abdominis plane block (TAP) was proposed as an effective measure as part of multimodal analgesia in abdominal surgery, including $\mathrm{PVH}$ plastic surgery. The essence of this procedure is to introduce a local anesthetic into the space between the internal oblique and transverse muscles of the abdomen, where there are branches of sensitive thoracolumbar nerves that innervate the anterolateral parts of the anterior abdominal wall. Prolonged liposomal forms of local anesthetics (most often bupivacaine) or prolonged catheter administration are used to prolong the duration of the blockade [58]. To ensure the effectiveness of the TAP-block procedure, a clear positioning of the needle (or catheter) in the intermuscular space is required. To do this, the most commonly used needle insertion under the control of ultrasound [59]. But it is possible to do this under the control of direct visualization [60]. The use of TAP-block contributed to reduce the duration of inpatient treatment and decrease the dose of opioids in the 1 st and 2 nd postoperative days compared with patients who received epidural analgesia [61]. A meta-analysis of 10 controlled studies found that TAP-block was no less effective than epidural blockade in children and adults, but could lead to hypotension [62]. Instead of the TAP-block, ilio-inguinal-iliohypogastric block was also proposed, which proved to be even more effective in terms of the effectiveness of analgesia 6 and 8 hours after surgery [63]. This was confirmed by another study in which the procedures were performed under ultrasound control and use of $0.25 \%$ bupivacaine at a dosage of $0.3 \mathrm{ml} / \mathrm{kg}$ of body weight [64].

Reducing the severity of the systemic inflammatory response, both in «open» and laparoscopic interventions, contributes to intra-abdominal administration during surgery of drugs that can reduce the severity of post-traumatic inflammation. Attention is drawn to the possibility of its modeling by blocking peripheral glutamatergic receptors $[65,66]$. The work of El-Gaby S.S. and Mohamed S.S. (2017) showed that intra-abdominal administration during laparoscopic intervention of ketamine, which has antagonism with respect to N-methyl-D-aspartate receptors, leads to a decrease in the severity of the systemic inflammatory response with a decrease in serum IL-6 in the postoperative period compared to the control group [67]. It should be noted that $\mathrm{N}$-methyl-D-aspartate (glutamate) receptors are localized not only in the central nervous system, but are present in the smooth muscles of the stom- ach and intestines, so their block not only reduces pain, but also reduces the severity of inflammation [68].

In addition, the use of glucocorticoids has been shown to be an effective way to reduce the severity of systemic inflammatory reactions. In particular, a randomized double-blind clinical study found that a single administration of $125 \mathrm{mg}$ of methylprednisolone immediately before surgery (during premedication) resulted in a significant reduction in C-reactive protein concentration, as well as a significant reduction in postoperative pain during activity and cough [69]. Another interesting method of reducing postoperative pain is the intra-abdominal administration of local anesthetics. H.M. Sultan et al. (2015) found a good effect of irrigation of the abdominal cavity with a solution of xylocaine and its introduction after surgery through a special port [70]. A similar result was obtained in another study [72]. N.W. Shady et al. (2018) used abdominal irrigation with a local anesthetic during laparoscopic surgery in patients with gynecological pathology [72]. Another promising way to increase the effectiveness of analgesia is patient-based protocols, which provide for the appointment of analgesics depending on the genetic characteristics of patients - the expression of genes that determine the effectiveness of narcotic and non-narcotic drugs [73].

The results of many studies show that in conditions of multimodal analgesia, in addition to reducing the intensity of postoperative pain, the restoration of respiratory function, stabilization of central hemodynamics and normalization of IAP accelerate, renal vascular resistance decreases and renal blood flow increases $[22,74,75]$. The use of perioperative multimodal analgesia under the ERAS protocol has reduced opioid use on the day of surgery and on the 1 st and 2nd postoperative day [76]. ERAS protocols have been recognized as effective in colorectal surgery, surgical hepatology, gastrectomy, pancreatic surgery, including in the elderly and senile, and in emergency surgery [77].

In recent years, there have been increasing reports of the effectiveness of individual measures or comprehensive accelerated recovery programs in the surgical treatment of ventral hernias. In the surgical treatment of PVH, these programs included preoperative prescription of glucocorticoids, daily evaluation of criteria for discharge and restoration of bowel function using chewing gum and cleansing enema on the second day after surgery [78], multimodal strategy of perioperative analgesia, 
use of oral opioid receptor blockers and early enteral nutrition [79]. Multimodal analgesia, early mobilization, and accelerated recovery of bowel function with the use of alvimopan in the surgical treatment of ventral hernias have led to a reduction in the duration of inpatient treatment by $15 \%$ [39]. Another study found that enhanced recovery programs helped to restore bowel function earlier and reduce the incidence of wound complications [80].

The introduction of multimodal anesthesia with extensive use of ibuprofen and acetomiphene significantly reduced the need for opioids after ventricular hernia hernioplasty [53]. Significantly reduced the intensity of postoperative pain, accelerated the recovery of intestinal function and reduced the duration of hospitalization with alvimopan, early nutrition and multimodal analgesia with an emphasis on intraoperative nerve block long-acting anesthetic (Exparel) [36]. Some measures that correspond to emergency surgery programs were also proved to be effective. Thus, in the hernioplasty of giant $\mathrm{PVH}$, the prescription of high doses of glucocorticoids before surgery helped to reduce the intensity and duration of pain, nausea and weakness. The duration of inpatient treatment decreased from 5.5 days to 3.0 days $(\mathrm{p}=0.003)$, but the frequency of complications and re-hospitalizations did not change [81]. Similar results were obtained in patients undergone surgery for large PVH [82].

But the data obtained are far from unambiguous. Thus, a study C. Harryman et al. (2020) did not find a significant effect of the strategy of enhanced recovery on the duration of hospitalization and the cost of expanses in patients with open hernioplasty of ventral hernias, although the authors note a faster recovery of intestinal function and reducing the incidence of wound complications [83]. According to a study by J. Colvin et al. (2019), the introduction of the ERAS protocol of a patient with ventral hernia did not affect the duration of hospitalization, there was only a tendency to reduce the duration of the required analgesia [6]. Some studies found no benefit on the use of epidural anesthesia in surgical treatment. In contrast, in the group of patients who received epidural an-

Conflict of interest: none declared

Financial support: none declared

\section{REFERENCES}

1. Weiser TG, Haynes AB, Molina G, Lipsitz SR, Esquivel MM, Uribe-Leitz T, et al. Size and distribution of the global volume of surgery in 2012. Bull World Health Organ. 2016;94(3):201-9F.

2. Global patient outcomes after elective surgery: prospective cohort study in 27 low-, middle- and high-income countries. BJA. 2016;117(5):601-9 esthesia, the duration of inpatient treatment of $\mathrm{PVH}$ was increased; there were complications of epidural anesthesia, and the intensity of postoperative pain was higher [84]. J. Jurt et al. (2017) found that along with the use of a nasogastric tube and drainage of the abdominal cavity, the frequency of complications was increased by intraoperative epidural anesthesia [24].

A recent systematic review and meta-analysis of 5 studies examining the results of the ERAS protocol in patients undergoing anterior abdominal wall reconstruction found no effect on recurrence rates and the incidence of wound complications, but a reduction in hospital stay duration [85]. According to the results of another systematic review and meta-analysis of 5 non-randomized studies, no other positive effect was found except for a reduction in the duration of hospitalization. Patients with enhanced recovery did not show a reduction in complications, bowel recovery time, opioid withdrawal time, urinary catheter removal time, restoration of normal nutrition, and readmission rates compared with controls [86].

\section{CONCLUSIONS}

The development and implementation of measures to prevent postoperative complications is a very important component of surgical treatment, especially in patients with extensive surgical interventions. Ventral hernia surgery, in particular large postoperative hernias, carries the risk of both systemic and local complications, which are the cause of long-term rehabilitation, recurrence of hernias and fatal consequences. In this aspect, the most promising are comprehensive FTS and ERAS strategies, which consist of various measures during the preparation for the surgery, during the surgery and in the postoperative period.

The results of research on the effectiveness of enhanced recovery programs in herniology are ambiguous. Issues remain about their feasibility depending on the location of the hernia, its size, method and scope of surgery, in the presence of comorbidity/or when performing simultaneous operations.
3. Ansari D, Gianotti L, Schröder J, Andersson R. Fast-track surgery: procedure-specific aspects and future direction. Langenbeck's Arch Surg. 2013;398(1):29-37.

4. Rodrigues G, Ravi Ch, Prabhu R. Fast-track surgery: A new concept of perioperative management of surgical patients. $J$ Health Specialties. 2013;1(3):114-21. 
5. Simsek T, Simsek HU, Cantürk NZ. Response to trauma and metabolic changes: posttraumatic metabolism. Ulus Cerrahi Derg (Turk J Surg). 2014;30(3):153-9.

6. Colvin J, Rosen M, Prabhu A, Rosenblatt S, Petro C, Zolin Samuel, et al. Enhanced recovery after surgery pathway for patients undergoing abdominal wall reconstruction. Surgery. 2019; 166(5):849-53.

7. Stojanovic MD, Markovic DZ, Vukovic AZ, Dinic VD, Nikolic AN, Maricic TG, et al. Enhanced recovery after vascular surgery. Front Med (Lausanne). 2018;5:2.

8. Orenstein SB, Martindale RG. Enhanced recovery pathway for complex abdominal wall reconstruction. Plast Reconstr Surg. 2018;142(3 Suppl):133S-41S.

9. Cox TC, Pearl JP, Ritter EM. Rives-Stoppa incisional hernia repair combined with laparoscopic separation of abdominal wall components: a novel approach to complex abdominal wall closure. Hernia. 2010;14(6):561-7.

10. Ortiz J, Wang S, Elayda MA, Tolpin DA. Preoperative patient education: can we improve satisfaction and reduce anxiety? Rev Bras Anestesiol. 2015;65(1):7-13.

11. Hartmann CEA, Ko LWL, Jones GJ, Nathwani D. Preoperative patient education as tool to improving post-operative patient recovery. Int J Surg. 2013;11(8):668.

12. Fink Ch, Diener MK, Bruckner T, Müller G, Paulsen L, Keller M, et al. Impact of preoperative patient education on prevention of postoperative complications after major visceral surgery: study protocol for a randomized controlled trial (PEDUCAT trial). Trials. 2013;14:271.

13. Slim K, Standaert D. Enhanced recovery after surgical repair of incisional hernias. Hernia. 2020;24(1):3-8.

14. Vaĭnsteĭn SG, Zhulkevich IV, Dubkin MS, Cherno NK. Food fibers as modifiers of homeostasis in patients with diabetes mellitus (in Russian). Ter Arkh. 1987;59(11):29-31.

15. Ball L, Pelosi P. Intraoperative ventilation and postoperative respiratory assistance. Brit J Anaesth Educ. 2017;17(11):357-62.

16. Futier E, Godet T, Millot A, Constantin JM, Jaber S. Mechanical ventilation in abdominal surgery. Ann Fr Anesth Reanim. 2014; 33(7-8):472-5.

17. Karcz M, Papadacos PJ. Respiratory complications in the postanesthesia care unit: A review of pathophysiological mechanisms. Can J Respir Ther. 2013;49(4):21-9.

18. Shyam DC, Rapsang AG. Inguinal hernias in patients of 50 years and above. Pattern and outcome. Rev Col Bras Cir. 2013;40(5):374-9.

19. Nanavati AJ, Prabhakar S. A comparative study of 'fast-track' versus traditional peri-operative care protocols in gastrointestinal surgeries. J Gastrointest Surg. 2014;18(4):757-67.

20. Cheng Y, Lu J, Xiong X, Wu S, Lin Y, Wu T, et al. Gases for establishing pneumoperitoneum during laparoscopic abdominal surgery. Cochrane Database Syst Rev. 2013;1:CD009569.

21. Ueda H, Hoshi T. Functional residual capacity increase during laparoscopic surgery with abdominal wall lift. Rev Bras Anestesiol. 2017;67(3):284-7.

22. McGlory G, Davis AE, Kirksey KM. Multimodal analgesia in critical care. Nurs Crit Care. 2018;13(2):18-23.

23. Sylla P, Kirman I, Whelan RL. Immunological advantages of advanced laparoscopy. Surg Clin North Am. 2005;85(1):1-18.

24. Jurt J, Slieker J, Frauche P, Addor V, Solà J, Demartines N, Hübner M. Enhanced recovery after surgery: can we rely on the key factors or do we need the bel ensemble? World J Surg. 2017; 41(10):2464-70.

25. Güenaga KF, Matos D, Wille-Jørgensen P. Mechanical bowel preparation for elective colorectal surgery. Cochrane Database Syst Rev. 2011;(9):CD001544.

26. Itou K, Fukuyama T, Sasabuchi Y, Yasuda H, Suzuki N, Hinenoya H, et al. Safety and efficacy of oral rehydration therapy until $2 \mathrm{~h}$ before surgery: a multicenter randomized controlled trial. J Anesthesiol. 2012;26(1):20-7.

27. Goudar BV, Kalburgi EB, Giraddi HL, Karikazi MA. Early removal versus conventional removal of nasogastric tube after abdominal surgery: a prospective randomized controlled study. Int J Surg. 2017;4(1):229-32.

28. Jangjoo A, Mohammadipoor F, Fazel A, Bahar MM. Aliakbarian M, Nooghabi MJ. The role of nasogastric intubation on postoperative gastrointestinal function in patients with obstructive jaundice. Indian $\mathrm{J}$ Surg. 2012;74(5):376-80.

29. Guerra F, Giuliani G, Coletta D, Boni M, Rondelli F, Bianchi PP, et al. A meta-analysis of randomized controlled trials on the use of suction drains following rectal surgery. Dig Surg. 2018;35(6):482-90.

30. Chauhan HR, Vaishnav UG. A comparative study to evaluate the outcome of routine use of drain verses no drain after laparoscopic cholecystectomy: a tertiary care teaching center experience. Int Surg J. 2016;3(1):330-5.

31. Messager M, Sabbagh C, Denost Q, Regimbeau JM, Laurent C, Rullier $\mathrm{E}$, et al. Is there still a need for prophylactic intra-abdominal drainage in elective major gastro-intestinal surgery? J Visc Surg. 2015;152(5):305-13.

32. Bajwa RS, Brar N. A prospective randomized controlled study: early enteral nutrition following gastrointestinal surgery. Int Surg J. 2017;4(10):3249-56.

33. Dorai D, Kumar LK, Chitra T, Prasanna G. Effects of early enteral nutrition on patients after emergency and elective gastrointestinal surgery. Int Arch Integrated Med (IAIM). 2016;3(8):1-10.

34. Tu CP, Tsai CH, Tsai CC, Huang TS, Cheng SP, Liu TP. Postoperative lleus in the Elderly. Int J Gerontol. 2014;8(1):1-5.

35. Vaĭnshteĭn SG, Masik AM, Zhulkevich IV. Food fiber - research results and outlook (in Russian). Vopr Pitan. 1988 Nov-Dec;(6):8-12.

36. Fayezizadeh M, Petro CC, Rosen MJ, Novitsky YW. Enhanced recovery after surgery pathway for abdominal wall reconstruction: pilot study and preliminary outcomes. Plast Reconstr Surg. 2014; 134(4 Suppl 2):151S-9S.

37. Radadia KD, Farber N, Tabakin A, Milas L, Jang T. Effect of alvimopan on gastrointestinal recovery and length of hospitalization after retroperitoneal lymph node dissection for testicular cancer. J Clin Oncol. 2017;35(6) Suppl:424.

38. Nair A. Alvimopan for post-operative ileus: What we should know? Acta Anaesthesiol Taiwan. 2016;54(3):97-8.

39. Ueland W, Walsh-Blackmore S, Nisiewicz M, Davenport DL, Plymale MA, Plymale $M$ et al. The contribution of specific enhanced recovery after surgery (ERAS) protocol elements to reduced length of hospital stay after ventral hernia repair. Surg Endosc. 2020;34(10):4638-44.

40. Bonventre S, Inviati A, Di Paola V, Morreale P, Di Giovanni S, Di Carlo $P$, et al. Evaluating the efficacy of current treatments for reducing postoperative ileus: a randomized clinical trial in a single center. Minerva Chir. 2014;69(1):47-55.

41. Ge W, Chen G, Ding YT. Effect of chewing gum on the postoperative recovery of gastrointestinal function. Int J Clin Exp Med. 2015; 8(8):11936-42.

42. Veelo DP, van Berge Henegouwen MI, Ouwehand KS, Geerts BF, Anderegg MC, van Dieren S, et al. Effect of goal-directed therapy on outcome after esophageal surgery: A quality improvement study. PLoS One. 2017;12(3):e0172806.

43. Voldby AW, Brandstrup B. Fluid therapy in the perioperative setting - a clinical review. J Intensive Care. 2016;4:27.

44. Hodgson CL, Capell E, Tipping CJ. Early mobilization of patients in intensive care: organization, communication and safety factors that influence translation into clinical practice. Crit Care. 2018;22(1):77.

45. Tipping CJ, Harrold M, Holland A, Romero L, Nisbet T, Hodgson CL. The effects of active mobilisation and rehabilitation in ICU on mortality and function: a systematic review. Intensive Care Med. 2017;43(2):171-83.

46. Morris PE, Berry MJ, Files DC, Thompson JC, Hauser J, Flores L, et al. Standardized rehabilitation and hospital length of stay among patients with acute respiratory failure: a randomized clinical trial. JAMA. 2016;315(24):2694-702.

47. Zhang L, Xu X. Therapeutic management of postoperative ileus. Transl Surg. 2017;2(2):50-3.

48. Nair AS. Management of opioid induced postoperative ileus: the current scenario. Anaesth Pain Intensive Care. 2016;20(4):380-2. 
49. Gan TJ, Robinson SB, Oderda GM, Scranton RS, Pepin J, et al. Impact of postsurgical opioid use and ileus on economic outcomes in gastrointestinal surgeries. Curr Med Res Opin. 2015;31(4):677-86.

50. Swift A. Non-opioid analgesia is as effective as opioid management in acute pain and supports a change in prescribing practice to help address the «opioid epidemic». Evid Based Nurs. 2018;21(2):50.

51. White PF. What are the advantages of non-opioid analgesic techniques in the management of acute and chronic pain? Expert Opin Pharmacother. 2017;18 (4):329-33.

52. American Society of Anesthesiologists Task Force on Acute Pain Management. Practice guidelines for acute pain management in the perioperative setting: An updated report by the American Society of Anesthesiologists Task Force on Acute Pain Management. Anesthesiology. 2012;116(2):248-273.

53. Peterman DE, Knoedler BP, Ewing JA, Carbonell AM, Cobb WS, Warren JA. Implementation of an evidence-based protocol significantly reduces opioid prescribing after ventral hernia repair. Am Surg. 2020;86(11):1602-6.

54. Gelman D, Gelmanas A, Urbanaitė D, Tamošiūnas R, Sadauskas S, Bilskienè $D$ et al. Role of Multimodal Analgesia in the Evolving Enhanced Recovery after Surgery Pathways. Medicina (Kaunas). 2018;54(2):20.

55. Memtsoudis SG, Poeran J, Zubizarreta N, Cozowicz C, Mörwald EE, Mariano ER, et al. Association of multimodal pain management strategies with perioperative outcomes and resource utilization: a population-based study. Anesthesiology. 2018;128(5):891-902.

56. Wahal C, Kumar A, Pyati S. Advances in regional anaesthesia: A review of current practice, newer techniques and outcomes. Indian J Anaesth. 2018;62(2):94-102.

57. Bojaxhi E, Lee J, Bowers S, Frank RD, Pak SH, Rosales A, et al. Paravertebral blocks reduce the risk of postoperative urinary retention in inguinal hernia repair. Hernia. 2018;22(5):871-9.

58. Tsai H-C, Yoshida T, Chuang T-Y, Yang S-F, Chang C-C, Yao H-Y, et al. Transversus abdominis plane block: an updated review of anatomy and techniques. Biomed Res Int. 2017;2017:8284363.

59. Børglum J, Gögenür I, Bendtsen TF. Abdominal wall blocks in adults. Curr Opin Anaesthesiol. 2016;29(5):638-43.

60. Doble JA, Winder JS, Witte SR, Pauli EM. Direct visualization transversus abdominis plane blocks offer superior pain control compared to ultrasound guided blocks following open posterior component separation hernia repairs. Hernia. 2018;22(4):627-35.

61. Warren JA, Carbonell AM, Jones LK, Mcguire A, Hand WR, Cancellaro VA et al. Length of stay and opioid dose requirement with transversus abdominis plane block vs epidural analgesia for ventral hernia repair. J Am Coll Surg. 2019;228(4):680-6.

62. Baeriswyl M, Zeiter F, Piubellini D, Kirkham KR, Albrecht E. The analgesic efficacy of transverse abdominis plane block versus epidural analgesia: A systematic review with meta-analysis. Medicine (Baltimore). 2018;97(26):e11261.

63. Zhou Y, Chen M, Zhang Y, Zhou H, Yu X, Chen G. Ilioinguinal/ iliohypogastric nerve block versus transversus abdominis plane block for pain management following inguinal hernia repair surgery: A systematic review and meta-analysis of randomized controlled trials. Medicine (Baltimore). 2019;98(42):e17545.

64. Bhatia N, Sen IM, Mandal B, Batra A. Postoperative analgesic efficacy of ultrasound-guided ilioinguinal-iliohypogastric nerve block compared with medial transverse abdominis plane block in inguinal hernia repair: A prospective, randomised trial. Anaesth Crit Care Pain Med. 2019;38(1):41-5.

65. Moharari RS, Hodavi M, Pourfakhr P, Najafi A, Etezadi F, Khajavi MR. Evaluation of the postoperative analgesic efficacy of intraperitoneal ketamine compared with bupivacaine in laparoscopic cholecystectomy. Arch Anesthesiol Crit Care. 2016;2:146-9.

66. Gom HM, Abd Elhamid B. Intraperitoneal ketamine reduces postoperative analgesic requirement in morbidly obese patients: a controlled study. Enliven J Anesthiol Crit Care Med. 2015;3:5.

67. El-Gaby SS, Mohamed SS. Intraperitoneal ketamine attenuates the inflammatory reactivity associated with pneumoperitoneum. Res Opin Anesth Intensive Care. 2017;4(3):149-55.
68. Filpa V, Moro E, Protasoni M, Crema F, Frigo G, Giaroni C. Role of glutamatergic neurotransmission in the enteric nervous system and brain-gut axis in health and disease. Neuropharmacology. 2016;111(1):14-33.

69. Jensen KK, Brøndum TL, Leerhøy B, Belhage B, Hensler M, Arnesen $\mathrm{RB}$ et al. Preoperative, single, high-dose glucocorticoid administration in abdominal wall reconstruction: A randomized, double-blinded clinical trial. Surgery. 2020;167(4):757-64.

70. Sultan HM, Zaid NA, AlKafrawy AE. Evaluation of intraperitoneal and preincisional port site local anesthetic use in laparoscopic cholecystectomy. Menoufia Med J. 2015;28(1):5-10.

71. Al Kizwini GAM. Intra-peritoneal xylocaine spraying for postoperative pain control in laparoscopic cholecystectomy: a prospective study at Al-Yarmouk teaching hospital. Mustansiriya Med J. 2017;16(3):83-90.

72. Shady NW, Sallam HF, Ali SS, Abbas AM. Effect of intraperitoneal and incisional port site lidocaine on pain relief after gynecological laparoscopic surgery: A randomized controlled study. Middle East Fertil Soc J. 2018;23(1):63-7.

73. Senagore AJ, Champagne BJ, Dosokey E, Brady J, Steele SR, Reynolds HL et al. Pharmacogenetics-guided analgesics in major abdominal surgery: Further benefits within an enhanced recovery protocol. Am J Surg. 2017;213(3):467-72.

74. Graff V, Grosh T. Multimodal analgesia and alternatives to opioids for postoperative analgesia. APSF Journal. 2018;33(2):33-68.

75. Wick E, Grant MC, Wu CL. Postoperative multimodal analgesia pain management with nonopioid analgesics and techniques. A Review. JAMA Surg. 2017;152(7):691-7.

76. Warren JA, Stoddard C, Hunter AL, Horton AJ, Atwood C, Ewing JA, et al. Effect of multimodal analgesia on opioid use after open ventral hernia repair. J Gastrointest Surg. 2017;21(10):1692-99.

77. Liu G, Jian F, Wang X, Chen L. Fast-track surgery protocol in elderly patients undergoing laparoscopic radical gastrectomy for gastric cancer: a randomized controlled trial. Onco Targets Ther. 2016;9:3345-51

78. Jensen KK. Recovery after abdominal wall reconstruction. Dan Med J. 2017;64(3):B5349.

79. Majumder A, Fayezizadeh M, Neupane R, Elliott HL, Novitsky YW. Benefits of multimodal enhanced recovery pathway in patients undergoing open ventral hernia repair. J Am Coll Surg. 2016; 222(6):1106-15.

80. Stearns E, Plymale MA, Davenport DL, Totten C, Carmichael SP, Tancula CS, Roth JS. Early outcomes of an enhanced recovery protocol for open repair of ventral hernia. Surg Endosc. 2018; 32(6):2914-22.

81. Jensen KK, Brondum TL, Harling H, Kehlet H, Jorgensen LN. Enhanced recovery after giant ventral hernia repair. Hernia. 2016; 20(2):249-56.

82. Jensen KK, Dressler J, Baastrup NN, Kehlet H, Jørgensen LN. Enhanced recovery after abdominal wall reconstruction reduces length of postoperative stay: An observational cohort study. Surgery. 2019;165(2):393-7.

83. Harryman C, Plymale MA, Stearns E, Davenport DL, Chang W, Roth JS. Enhanced value with implementation of an ERAS protocol for ventral hernia repair. Surg Endosc. 2020;34(9):3949-55.

84. Prabhu AS, Krpata DM, Perez A, Phillips S, Huang L-C, Haskins IN et al. Is it time to reconsider postoperative epidural analgesia in patients undergoing elective ventral hernia repair?: An AHSQC Analysis. Ann Surg. 2018;267(5):971-6.

85. Lode L, Oma E, Henriksen NA, Jensen KK. Enhanced recovery after abdominal wall reconstruction: a systematic review and metaanalysis. Surg Endosc. 2020;35(2):514-23.

86. Sartori A, Botteri E, Agresta F, Gerardi C, Vettoretto N, Arezzo A, et al. Should enhanced recovery after surgery (ERAS) pathways be preferred over standard practice for patients undergoing abdominal wall reconstruction? A systematic review and meta-analysis. Hernia. 2020. 